\title{
Control Methods for a Teleoperated Endoscope Robot
}

\author{
Oğulcan Işıtman and Mehmet İsmet Can Dede \\ İzmir Institute of Technology, İzmir, Turkey, \\ ogulcanisitman@iyte.edu.tr \\ candede@iyte.edu.tr
}

\begin{abstract}
In this study, control of a endoscope robot for the pituitary gland surgery is presented. This co-worker robot has non-backdrivable actuation system with external brakes on the actuators. Since it is required to move in a constrained environment, which is the inside the nostrils in this case, modifications are required for the motion controller. In order to provide safe procedure, the maximum force and torque limits are defined for the real surgical case by using human cadaver head. By considering these limits and application specific requirements, a compliance controller is proposed and experimentally tested.
\end{abstract}

Keywords: compliant control, endoscope robot, minimally-invasive surgery, pituitary gland surgery

\section{Introduction}

The minimally invasive endoscopic pituitary gland tumor surgery is one of the challenging cases among the surgical robotics applications [1]. The pituitary gland controls several other hormone glands and it directly or indirectly affects the working mechanism of all organs in the human body. Any pathology constructed around the pituitary causes serious health problems. The cure for this type of tumors is performed either by opening the skull for an open-brain surgery or an endoscopic surgery through the nasal cavity.

One of the major problems encountered during this operation is that the surgeon has to use one hand to hold an endoscope throughout the surgery. Since the surgery takes 2-4 hours, the surgeon gets tired which decreases the efficiency of the operation. In usually, as assistant directs the endoscope while surgeon handles the other tools. However, this causes synchronization problems [2].

With the motivation of increasing efficiency of the procedure by giving third hand to the surgeon, a research project called "Robot-assisted endoscope control that can be controlled by the surgical tools (NeuRoboScope)" is initiated. In this project, a robotic co-worker is designed to manipulate the endoscope during the surgery alongside the surgeon. This co-worker can be directed by the surgeon both via teleoperation and via direct physical interaction.

The NeuRoboScope robot has a real-time teleoperation control capability which is composed of a wearable ring system that captures and transmits voluntary hand motions of the surgeon over a wireless communication channel to a 
slave system. Accordingly, the slave system processes the received data to generate velocity demands for the robot handling the endoscope. The endoscope robot in Figure 1, consists of two main parts which are passive serial arm and active parallel arm.

The active arm has 3 degree-of-freedom (DoF); 2 rotational and 1 translational [3].It is a remote center of motion kinematics based parallel manipulator and it contains DC motors with high-reduction ratio speed reducers and external brakes. The brakes are used to increase the safety in case of an emergency such as a power cut. Additionally, when the surgeon does not want to move the endoscope or the endoscope robot has already reached its predetermined torque/force limits due to the robot-tissue interaction, the brakes are activated to restrict the motion.

Since the robot moves inside a human nasal cavity, the motion is constrained and the system has to be comply with the environment in order not to apply excessive force to the soft tissue surrounding the endoscope. Therefore, the safety of procedure is one of the key points of the project. In addition to that, due to the existence of the brakes, the motion of the robot is not a continuous one which might result in instantaneous jumps of the endoscope motion at the time of motion initiation because the compressed tissue applies a load to the robot. Since the endoscope provides visual feedback to the surgeon, this kind of involuntary motion has to be avoided. Therefore, in this study, the motion control algorithm of such system is proposed while meeting the safety requirements.

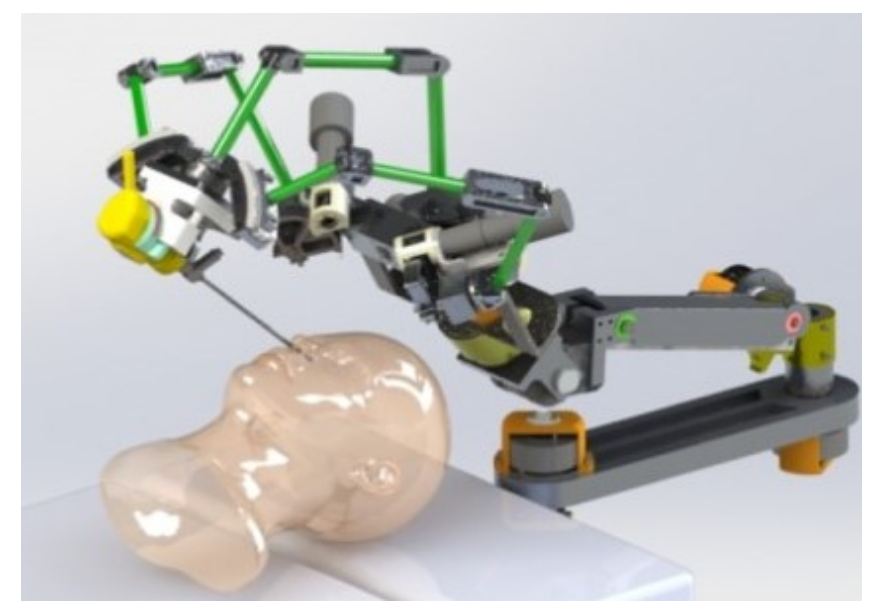

Fig. 1. 3D drawing of NeuRoboScope robot

In order to apply the proposed controller in a real surgical case, the force and moment limits are obtained to determine the limits of physical interaction and the results are presented in section 2 . In section 3 , the proposed algorithms are explained. Then, the experimental setup is described and results are provided. 
In the last section, the outcome of the study is discussed and conclusions are driven.

\section{Force and Moment Limit Tests}

In pituitary gland surgery, the endoscope handled by the robot is expected to interact with the nose tip and the nasal concha by making rotational motion. In addition to that, endoscope moves through the nasal cavity by making one-DoF translational motion. To avoid excessive contact force/moment to the environment and determine the required actuator for the system, it is required to obtain the maximum range of forces and moments applied by the surgeon during the surgery. Therefore, three experiment are designed for both part of the nasal tissue; nose tip and nasal concha. Measurements are acquired as surgeons used a mobile measurement device, which is introduced in [4], on a relatively fresh human cadaver head in ex-vivo tests.

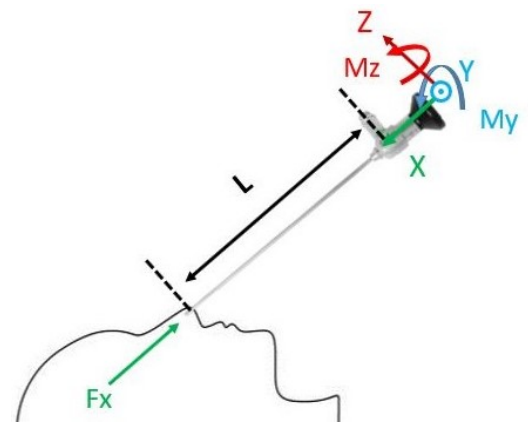

Fig. 2. Nose tip force and moment limits test scheme [6]

The first test is designed to measure the force and moments about two axes $(\mathrm{Y} \& \mathrm{Z})$ that are applied at the tip of the nose and the schematic representation of the test is given in Figure 2. In this test, the surgeon is asked to hold the device similar to the way endoscope is handled and apply the maximum force that can be applied during the surgery to the tip of the nose in order to mimic the limits of this surgery procedure. The procedure is repeated several times.

Test results are given in Table 1 indicating average values of five repetitions. The maximum values are determined as $\approx 2 N$ and $\approx 0.3 N m$ for the force $F_{y}$ and moment $M_{z}$ applied at tip of the nose.

In order to measure friction force during axial translation of the endoscope, another test is carried out and the results are also given in Table 1. In this test, the surgeon is asked to apply force to the tip of the nose to mimic a normal force applied to the surface and then, move the endoscope along the axial direction inside the nasal cavity by keeping the contact with the pivot point. Results show that, the axial movement of the endoscope results in significant force $(\approx 3 N)$ 
Table 1. Nasal concha tissue force and moment measurements

\begin{tabular}{|l|c|c|c|c|c|}
\cline { 2 - 6 } \multicolumn{1}{c|}{} & \multicolumn{4}{c|}{ Tip of the nose } & Friction Force \\
\cline { 2 - 6 } \multicolumn{1}{c|}{} & $\mathbf{F y}(\mathbf{m N})$ & $\mathbf{F z}(\mathbf{m N})$ & $\mathbf{M y}(\mathbf{m N m})$ & $\mathbf{M z}(\mathbf{m N m})$ & $\mathbf{F x}(\mathbf{m N})$ \\
\hline Average & 1727.14 & -210.7 & -73.14 & 303.62 & 2118.55 \\
\hline Absolute Max & 2033.9 & 472.7 & 136.6 & 353.8 & 3213.6 \\
\hline
\end{tabular}

which effects the selection of the actuator. The time series data of these tests could not be displayed in this paper due to space limitations.

The third test is realized in the nasal cavity. In this experiment, the surgeon is asked to apply force to the nasal concha without contacting the tip of the nose.

In these measurements, force is applied in different axes on a nasal concha. The obtained results are given in Table 2 which presents eight repetitions.

Table 2. Nasal concha tissue force and moment measurements

\begin{tabular}{|c|c|c|c|c|c|}
\hline Test & $\mathbf{F x}(\mathrm{mN})$ & Fy $(\mathrm{mN})$ & Fz $(\mathbf{m N})$ & My (mNm) & $\mathrm{Mz}(\mathrm{mNm})$ \\
\hline$\# 1$ & $-403,9$ & $-5063,5$ & 1274,6 & $-131,4$ & $-769,3$ \\
\hline$\# 2$ & $-338,9$ & $-2237,5$ & $-4184,1$ & 872,9 & $-489,4$ \\
\hline$\# \mathbf{3}$ & $-685,0$ & 2214,7 & 5841,9 & $-907,9$ & $-287,8$ \\
\hline$\# 4$ & 20,3 & 93,3 & $-546,7$ & 170,7 & 17,8 \\
\hline$\# 5$ & $-3125,1$ & 1771,6 & 11175,0 & $-1983,1$ & 386,9 \\
\hline$\# 6$ & 70,3 & 125,6 & 1770,9 & $-286,7$ & 14,2 \\
\hline$\# 7$ & $-1382,4$ & 2202,0 & 7494,8 & $-1229,0$ & 369,4 \\
\hline$\# 8$ & $-6220,0$ & 1365,4 & $-670,3$ & $-130,4$ & $-230,7$ \\
\hline
\end{tabular}

During the data acquisition process, the surgeon defined the applied forces as regular, maximum acceptable and excessive regarding his experiences in real surgery conditions. According to this evaluation, \# 1 and \#2 are defined as maximum but acceptable forces, \# 3 and 7 defined as a normal force however they do not occur often, \# 4 and \# 6 are in a regular force range and \# 5 defined as an excessive force that should not be applied by the endoscope.

\section{The Control Algorithm}

The control scenario of the endoscope holder robot starts by receiving the velocity demand. Surgeon initiates the generation of the velocity demand by pressing a foot pedal, then by using wearable ring system the voluntary hand motions are captured and transmitted to the slave system via a wireless communica- 
tion channel. The hand orientation is mapped to the desired angular and linear velocities of the endoscope in the predetermined axes [5].

Since the endoscope holder robot is a non-backdrivable system with a relatively high reduction ratio(1:905), external force/torque has a very small effect on the system. Hence, pure position control can be implemented. However, for direct drive systems, position control should be modified to reduce the effects of external force/torque.

Figure 3, represents a flowchart of possible control algorithms for both nonbackdrivable and backdrivable cases. Although the external torque has no significant effects on the non-backdrivable system, the existence of the brake and a torque limit due to the safety requirement requires application based modifications.

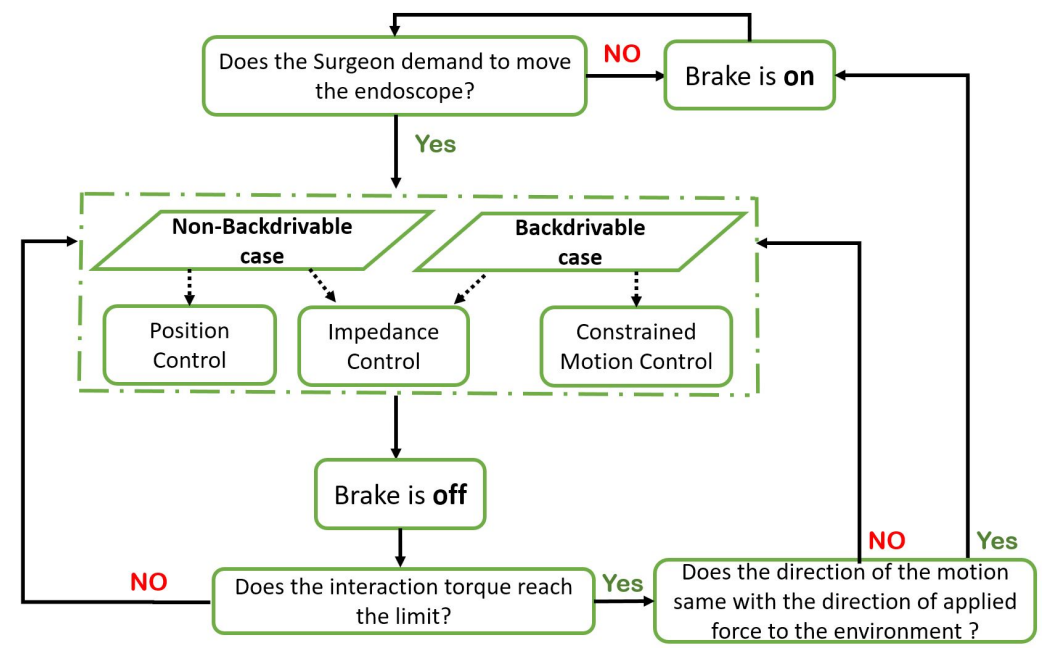

Fig. 3. Flowchart of the controller for the surgical scenario

The control procedure starts with the motion demand received from the surgeon by pressing to the foot pedal. Then the controller is activated and the brakes are released. If the interaction torque does not reach its limit value, then the motion of the endoscope continues with respect to the surgeon's demand. If the interaction occurs and the torque reaches its limit and the surgeon still demands to move the endoscope through soft tissue then the brakes are activated and the motion is restricted. If the surgeon demands to move the endoscope outside to the soft tissue then the brakes are released and the control is activated again.

The proposed controllers for the non backdrivable case are experimentally tested and discussed in the next section. For the backdrivable case, the constrained motion control is proposed however this case is the out of scope for this study since the actuation system is not a backdrivable one. 


\section{Experimental Results}

In order to test the proposed interaction control algorithm, a single DoF experimental setup is designed and presented in Figure 4.

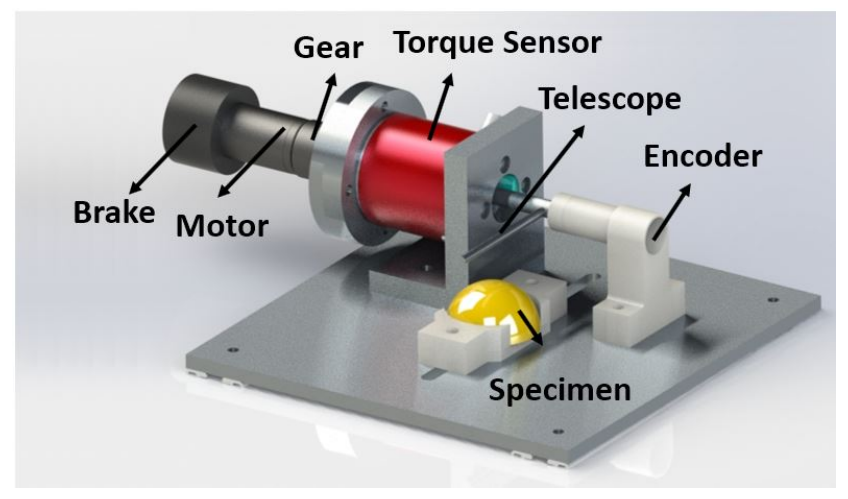

Fig. 4. The CAD model of the experimental set-up

To mimic the actuation system of the endoscope, a DC motor (Maxon RE25339155 ) with the planetary gearhead (Maxon GP26A) and a brake (Maxon Brake AB28) is used. A reaction type torque sensor (Futek TFF400) is assembled to the system in order to measure the applied external torque due to the interaction with a specimen that mimics the soft tissue. The angular position is measured by using an optical quadrature encoder (AEDA-3300AT). The part called telescope simulates the actual telescope on the endoscope. For running the controller and data acquisition purposes, MyRIO DAQ board is used in combination with the Labview programming environment. Initially, pure position controller is examined. The conditions related to the state of the brake are investigated. Another controller is designed to define a compliance between the soft tissue and the telescope of the endoscope.

\subsection{Pure Position Controller Studies}

When there is more than one DoF in a robotic system and the speed reduction ratio is high enough, a common control method is the independent joint controller. In this controller, each joint is controlled by a motion control algorithm disregarding the effects of other joints' motion [7]. conditions investigated when this controller is employed are the changes of the brake states. The system operates as follows: (1) the surgeon presses the foot pedal to initiate the velocity demand flow, (2) the brakes move from on state to off state, (3) the endoscope robot is moved relative to its current position by the issuing the velocity demands received from the surgeon, (4) surgeon is satisfied with the pose of the endoscope and releases the pedal, (5) the brakes move from off state to on state, 
(6) the last position of the joint is recorded for the next time that the brakes will be in on state to add the initial position information to the integral of the velocity demand, (7) the brakes can also move to the off state if the designated values of force/torques are exceeded.

The corresponding controller is represented in Figure 5 where $\theta_{e}$ and $K_{E}$ are the position and the stiffness of the environment, respectively. The velocity input, $\omega_{r}$, is provided by using an analog joystick. $\theta_{r}$ represents the generated relative position reference and $\theta_{m}$ is the measured position of the endoscope. The overall open loop transfer function, $G_{m}(s)$, is presented in 1 .

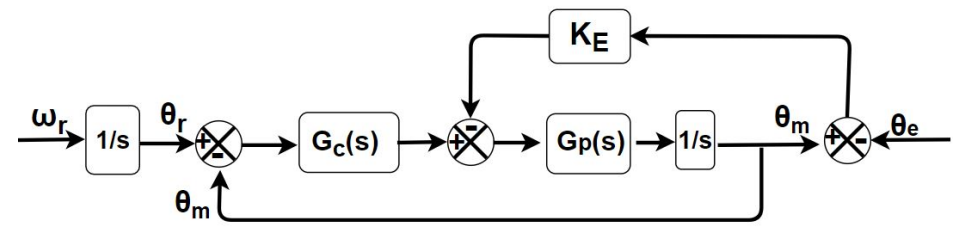

Fig. 5. Block diagram of position controller in a constrained environment

$$
G_{m}(s)=\frac{G_{c} G_{p}}{s\left(G_{c} G_{p}+K_{e} G_{p}+s\right)}
$$

In the figure, $G_{c}(s)$ and $G_{p}(s)$ represent the PD controller and transfer function of the robot, respectively.

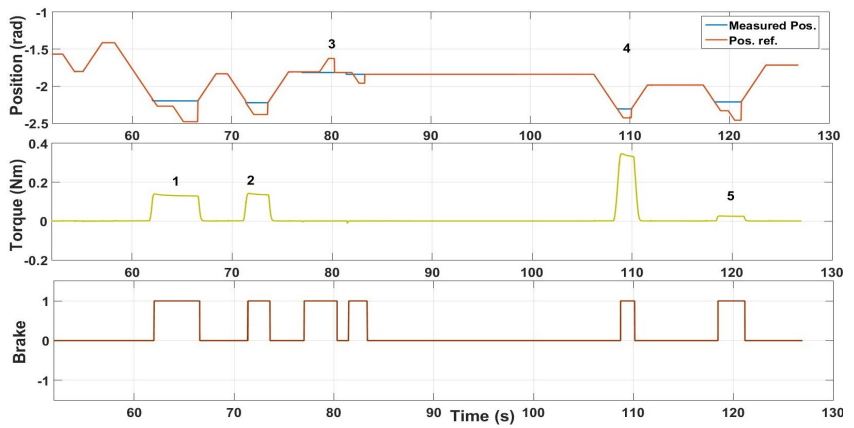

Fig. 6. Experimental results of the pure position control

The experimental results are presented in Figure 6. Various limits of interaction torques are tested. In situations 1 and 2, telescope reached the interaction torque limit $(0.1 \mathrm{Nm})$ and the brake is activated. Similar behavior is observed in situations 4 and 5 with the $0.3 \mathrm{Nm}$ and $0.01 \mathrm{Nm}$ torque limits, respectively. In situation 3 , the brake is activated externally, therefore the motion is restricted and reference signal starts to increase. When the brake is released, the reference signal is set to the measured position to prevent undesired movements. This working scheme is in accrodance with the aforementioned step (6). 
With this test, it is shown that a continuous operation is guaranteed even if the brake on/off states are activated by the surgeon or torque limits.

\subsection{Impedance Controller Studies}

By applying impedance controller, the dynamics of the physical interaction can be regulated by relating external torque acting on the telescope with its angular velocity [8].

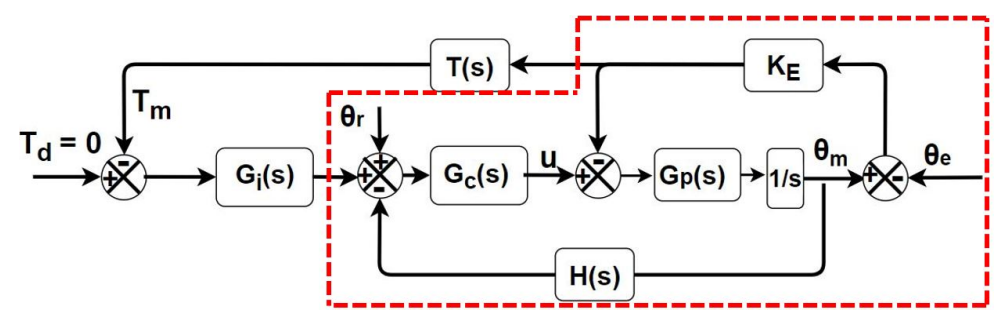

Fig. 7. Block diagram of the impedance controller

As in the previous controller case as velocity is the reference input, however, in order to design an impedance controller, controller scheme of the pure position controller is modified as presented in Figure 7, where the desired external torque, $T_{d}$, is defined as zero and the reference position input is considered as a disturbance. This approach allows to simplify the block diagram and selecting the impedance term's parameters. In this figure, $G_{i}(s)$ is the transfer function of the impedance term, $T_{m}$ is the measured interaction torque. $T(s)$ and $H(s)$ represent the transfer function of the torque sensor and the transfer function of the encoder, respectively. While determining the parameters of the impedance term, transfer functions inside dashed red line are taken as 1, by assuming that the inner loop of the controller runs much more faster than the outer loop.

Impedance term is defined as a mass-damper term and the corresponding transfer function is given in Equation 2.

$$
G_{i m p}=\frac{1}{m s^{2}+b s}
$$

The open-loop transfer function is re-organized to define damper parameter as an open loop gain which is given in Equation 3.

$$
G_{o, i m p}=\left(\frac{1}{b}\right) \frac{m s^{2}+K_{E}}{s}
$$

In the experiment, a silicone specimen is used to mimic the soft tissue. The stiffness of the silicone is estimated as $K_{E}=1.018 \mathrm{Nm} / \mathrm{rad}$ by following the estimation procedure defined in [4].

The mass is defined as $m=0.005 \mathrm{~kg}$ then 2 different damper gains are selected as $b_{1}=0.03 \mathrm{Nms} / \mathrm{rad}$ and $b_{2}=0.5 \mathrm{Nms} / \mathrm{rad}$ which correspond to the damping ratios of $(\zeta) 0.21$ and 1 , respectively. 
The experimental results of the impedance controller are represented in Figure 8 . In the figure, the horizontal dashed line (at $-2 \mathrm{rad}$ ) represents the location of the specimen. In situation 1, the interaction is initiated and consequently, the measured interaction torque starts to increase. The difference between the reference position and the measured position is compensated by the impedance term. Therefore, the interaction torque increased. Due to the selected damping ratio $(\zeta=0.21)$, a damped oscillation is observed when the interaction has occurred.

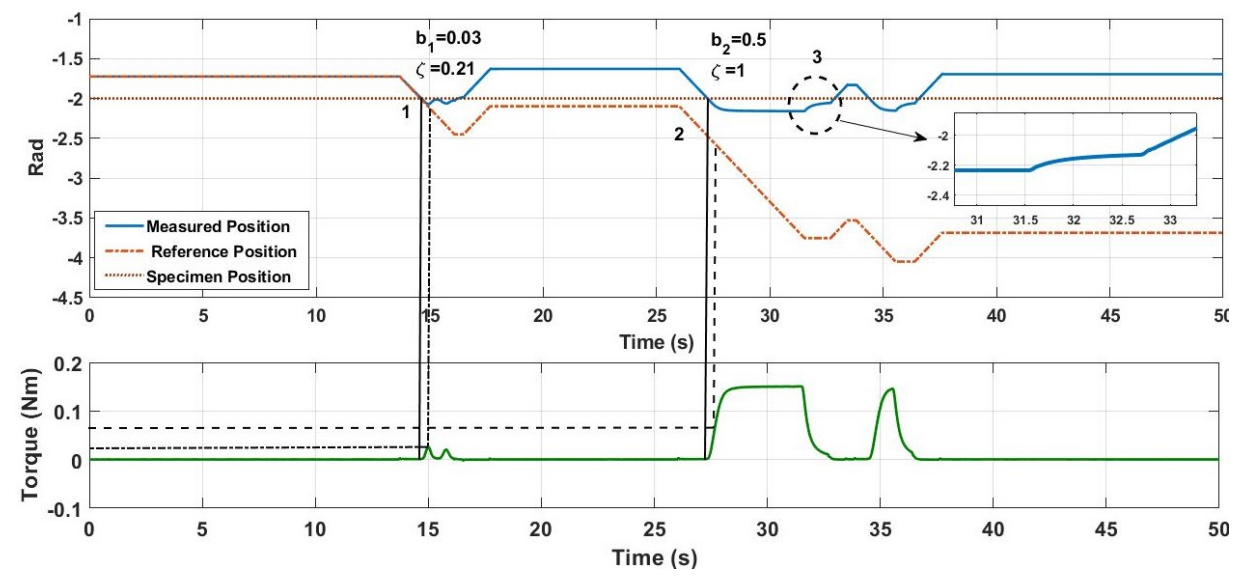

Fig. 8. Experimental results of the impedance controller

In situation 2, the interaction has occurred but this time the damping ratio is used as 1 . With this new parameter, the controller moves the endoscope to penetrate the tissue without any oscillation. However, for the same amount of the position input, the interaction torque value is increased with respect to the situation 1 . This can be observed by the vertical lines in the figure. According to the selection of the damping ratio, the open loop gain for situation 2 is smaller than situation 1 and thus, this results in a smaller modification of the position for the same amount of interaction forces. As a result of this, for the same type of motion demand higher interaction torques are obtained in situation 2 with the damping ratio of 1 .

In situation 3 which is presented in dashed circle, the velocity reference is set to zero to keep its position, consequently the endoscope started to move out slowly from the tissue even if the actuation system has a large reduction ratio which minimizes the backdrivability.

The difference between the reference position and the measured position is increased due to the integral effect of the impedance term. This difference is not the error of the controller, it is compensated by the impedance term. The actual input from the surgeon is the velocity. Therefore, the controller is capable of following the reference velocity even though position difference is increased. 


\section{Conclusions and Discussions}

In this study, two control algorithms are proposed for the NeuRoboScope project's endoscope robot. These algorithms are tested in 1-dof experimental set-up.

In NeuRoboScope case, the pure position controller can be used due to the high reduction ratio of the gears used in the actuation system (1:905). The external torque has to be measured in order to activate the brakes to avoid the application of excessive forces to the soft tissue. This controller allows controlling the position without being affected by the external torques due to the interaction with the soft tissue. In order to implement this controller for a real surgical case, the torque limits for the the human nasal cavity are identified experimentally by using a human cadaver head.

If the application requires to control the dynamics between the robot and the soft tissue, impedance control can be applied. By using the environment model, proper impedance terms can be selected via adjusting the trade-off between oscillatory behaviour and external torque limits. This controller also requires a torque sensor. By controlling the dynamics of the interaction, torque limit violation can be avoided without activating the brakes.

The future study of this work includes the use of backdrivable actuation systems and implementation of these controllers in the actual robot.

Acknowledgement This work is supported by The Scientific and Technological Research Council of Turkey via grant number 115E726.

\section{References}

1. Schneider, John S., et al. "Robotic surgery for the sinuses and skull base: What are the possibilities and what are the obstacles?." Current opinion in otolaryngology \& head and neck surgery 21.1 (2013): 11.

2. Burgner, Jessica, et al. "A telerobotic system for transnasal surgery." IEEE/ASME Transactions on Mechatronics 19.3 (2014): 996-1006.

3. Yaşır, Abdullah. Design of a 2R1T mechanism with remote center of motion for minimally invasive transnasal surgery applications. MS thesis. Izmir Institute of Technology, 2018.

4. Işıtman, O., et al. "Viscoelastic modeling of human nasal tissues with a mobile measurement device." New Trends in Medical and Service Robotics. Springer, Cham, 2019. 216-224.

5. Ateş, Gizem, Ronny Majani, and Mehmet İsmet Can Dede. "Design of a teleoperation scheme with a wearable master for minimally invasive surgery." New Trends in Medical and Service Robotics. Springer, Cham, 2019. 45-53.

6. Işıtman, Ŏ̆ulcan. Compliant control of a teleoperated endoscope robot. MS thesis. Izmir Institute of Technology, 2018.

7. Sciavicco, Lorenzo, and Bruno Siciliano. Modelling and control of robot manipulators. Springer Science \& Business Media, 2012.

8. Hogan, Neville. "Impedance control: An approach to manipulation." American Control Conference, 1984. IEEE, 1984. 